\title{
Creep and damage in shells of revolution under cyclic loading and heating
}

\author{
D. Breslavsky ${ }^{\mathrm{a}, *}$, O. Morachkovsky ${ }^{\mathrm{b}}$, O. Tatarinova ${ }^{\mathrm{a}}$ \\ a Department of Control Systems and Processes, National Technical University "Kharkiv Polytechnic Institute", Kharkiv, Ukraine \\ ${ }^{\mathrm{b}}$ Department of Theoretical Mechanics, National Technical University “Kharkiv Polytechnic Institute", Kharkiv, Ukraine
}

\section{A R T I C L E I N F O}

Available online 20 February 2014

Keywords:

Creep

Damage

Shell of revolution

Cyclic loading and heating

Asymptotic expansions

FEM code

\begin{abstract}
A B S T R A C T
Creep of cyclically loaded thin shells of revolution and their fracture due to creep and fatigue mechanisms are studied. Creep-damage equations for steels and nickel-based alloys are built by the use of scalar damage parameter. Constitutive equations were derived using the method of asymptotic expansions and averaging over a period of cyclic loading. The cases of fast and slow varying of temperature and loading are regarded. General problem statement and method for solution of creep problems at cyclic loading are presented. Strain-stress state in shell structures is determined by the use of homemade FEM creep-damage code, where the finite element of conical shell is used. Results of creep-damage problem for conical panel are discussed.
\end{abstract}

c) 2014 Elsevier Ltd. All rights reserved.

\section{Introduction}

Cyclic loading and heating are often met in industrial applications. As a rule, at elevated temperatures they essentially aggravate the working conditions of structural elements because of increasing of creep strain rate as well as decreasing of time to fracture values. Because of the long duration and high costs of experimental investigations of structural elements; their numerical simulations are necessary. Despite the fact that solutions of linear problems can be obtained numerically by the use of FEM software, modeling of non-linear problems meet considerable difficulties. One of them is adequate formulation of constitutive equations, especially for complex cases of deformation processes like cyclic loading. The case of joint action of static and cyclically varying loading have long attracted the attention of scientists and engineers. The more general case of stresses which exceed the yield limit has been studied in detail [1-6]. The interest to such phenomenon continues unabated and interaction between low-cycle fatigue and creep effects for the cases of cyclic varying of loads and temperatures attracts the attention of researchers over the last decade [7-10].

However, operational conditions of many structural elements which work in aviation, power and chemical industry are designed so that arising stresses do not exceed yield limit. This fact allows the use of simplified approaches, one of the most effective is averaging of resulting curves of strain growth and damage

\footnotetext{
* Corresponding author. Tel.: + 380577076058 .

E-mail address: brdm@kpi.kharkov.ua (D. Breslavsky).
}

accumulation. If the number of cycles is high, the changes of these parameters through a cycle can be regarded as negligible, but the cyclic varying of stresses and temperatures influences on the rates of strain and damage parameter. For the case of cyclic variation of temperatures or stresses the approach connected with averaging of resulting creep strain curves has been shown to be effective [11-15]. Such clear physical procedure got its mathematical justification by the way of using the methods of asymptotical expansions and averaging over a period of stress or temperature variation [15-17].

Present investigations which have been done in this direction were focused on particular cases of cyclic creep-damage problems. The phenomenon of so-called dynamic creep [12], when cyclic variation of stresses due to forced vibrations is imposed on the static stress component has been investigated for plane creep problems [16]. Further interaction between damage accumulation from dynamic creep process and high cycle high temperature fatigue has been analyzed for thin shells of revolution in [17]. The situations of slow variation of stresses and temperatures were studied in $[18,19]$.

However, a certain number of structural elements is loaded and heated by complex programs. In addition their forced vibrations cannot be completely suppressed. That is why the formulation of the generalized approach to description of creep-damage processes allowing consideration of complex programs of loading and heating can be regarded as useful for engineering applications.

The main goal of this paper is to discuss the derivation method for creep problems with complex cyclic loading or heating programs. The examined problem can be considered as an 
example of the application of this technique. With this example in mind the other practical problems with different types of cyclic programs can be solved. However, the most common cases are considered.

The paper contains the generalized constitutive model in which the list of possible effects related to cyclic character of loading and heating is considered. The method of solution is presented first for general 3D case and further for thin shells of revolution. FEM approach with time numerical integration was used.

\section{Constitutive model for different cases of cyclic loading}

The mostly common case which includes the cycles of loading/ unloading and heating/cooling is regarded. Let us first regard at the behavior of the material at uniaxial stress state. Results will be further generalized to the complex stress state.

It is well known that mechanical behavior of the specimen subjected by cyclic loading significantly depends upon its frequency. The cyclic creep-damage processes in a solid which are originated by the action of temperature field can be divided into the action of low or high cycle loading or heating.

Equations for rates of creep strain $c$ and damage parameter $\omega$ for the uniaxial loading that are used in the Bailey-Norton and Rabotnov-Kachanov [20] forms:

$\dot{c}=B \frac{(\sigma)^{n}}{(1-\omega)^{k}}, \quad \dot{\omega}=D \frac{(\sigma)^{r}}{(1-\omega)^{l}}$

where $B, n, k, D, r$, and $l$ are the material constants.

Let us limit by this constitutive model.

For all cases of cyclic loading total stress in the specimen is presented as a sum of constant part $\sigma$ and varying part $\sigma^{1}: \bar{\sigma}=\sigma+\sigma^{1}$. Just the same the temperature has constant part $T$ and varying part $T^{1}: \bar{T}=T+T^{1}$.

Firstly let us regard the quasi static creep processes with long periods more than $1 \mathrm{~s}$. Generally they can be presented as polyharmonic with the global period $T_{\sigma}$ :

$\bar{\sigma}=\sigma+\sigma^{1}=\sigma\left(1+\sum_{k=1}^{\infty} M_{k} \sin \left(\frac{2 \pi k}{T_{\sigma}} t+\gamma_{k}\right)\right)$

At first we consider that temperature has the constant value $T$.

We expand creep strain and the damage parameter into asymptotic series of the small parameter $\mu=T_{\sigma} / t_{*} \ll 1$ [21] and keep two terms of series:

$c \cong c^{0}(t)+\mu c^{1}(\xi), \quad \omega \cong \omega^{0}(t)+\mu \omega^{1}(\xi)$

where $c^{0}(t), \omega^{0}(t), c^{1}(\xi), \omega^{1}(\xi)$ are the functions which coincide with basic creep-damage process in slow (0) and fast (1) time scales.

Used truncated asymptotic series have direct physical meaning: the first term corresponds to slow motion whereas the second describes the motion through a cycle.

Two times are considered: slow time $t$ which varies from 0 to the time to rupture moment $t_{*}$; fast time $\tau=t / \mu$ or $\xi=\tau / T_{\sigma}$ $(0 \leq \xi \leq 1)$ [18]. After the substitution of Eq. (3) into Eq. (1) and further averaging $[21,22]$ over the period of stress variation we obtain the expressions of creep strain and the damage parameter on the time interval $(0 \leq \xi \leq 1)$ :

$\left\langle c^{0}(\xi)\right\rangle=\int_{0}^{1} c^{0}(t) d \xi \cong c^{0}(t), \quad\left\langle c^{1}(\xi)\right\rangle=\int_{0}^{1} c^{1}(\xi) d \xi \cong 0$,

$\left\langle\omega^{0}(\xi)\right\rangle=\int_{0}^{1} \omega^{0}(t) d \xi \cong \omega^{0}(t), \quad\left\langle\omega^{1}(\xi)\right\rangle=\int_{0}^{1} \omega^{1}(\xi) d \xi \cong 0$,

Substituting Eqs. (4) and (5) into Eq. (1) we obtain [18]:
$\dot{C}=B g_{n}\left(M_{k}\right) \frac{(\sigma)^{n}}{(1-\omega)^{m}}, \quad \dot{\omega}=D g_{r}\left(M_{k}\right) \frac{(\sigma)^{r}}{(1-\omega)^{1}}, \quad \omega(0)=\omega_{0}, \omega\left(t_{*}\right)=\omega_{*}$

$g_{n}\left(M_{k}\right)=\int_{0}^{1}\left(1+\sum_{k=1}^{\infty} M_{k} \sin (2 \pi k \xi)\right)^{n} d \xi$,

$M_{k}=\frac{\sigma^{a k}}{\sigma}, g_{r}\left(M_{k}\right)=\int_{0}^{1}\left(1+\sum_{k=1}^{\infty} M_{k} \sin (2 \pi k \xi)\right)^{r} d \xi$.

The main result here is following: we escaped from the necessity of the integration over the cycle and obtained averaged cyclic creep-damage laws. Here the functions $g_{n}\left(M_{k}\right)$ and $g_{r}\left(M_{k}\right)$ reflect the influence of cyclic character of loading.

If we consider more general low cycle process when not only stress varies due to law (2) but the temperature also:

$\bar{T}=T+T^{1}=T\left(1+\sum_{i=1}^{\infty} M_{i}^{T} \sin \left(\frac{2 \pi i}{T_{T}} t+\gamma_{i}^{T}\right)\right), \quad M_{i}^{T}=T_{i}^{a} / T$,

the creep-damage laws can be written in the following forms [20]:

$\dot{c}=B \frac{\bar{\sigma}^{n}}{(1-\omega)^{m}} \exp \left(-\frac{U_{c}}{R \bar{T}}\right)=B(\bar{T}) \frac{\bar{\sigma}^{n}}{(1-\omega)^{m}}$,

$\dot{\omega}=D \frac{\bar{\sigma}^{r}}{(1-\omega)^{l}} \exp \left(-\frac{U_{d}}{R \bar{T}}\right)=D(\bar{T}) \frac{\bar{\sigma}^{r}}{(1-\omega)^{l}}$

Here $U_{c}$ and $U_{d}$ are the constants which are equal to the values of activation energy in creep and creep damage processes, $R$ is the universal gas constant. In the remainder we use the notations $Q=U_{c} / R$ and $\bar{Q}=U_{d} / R$.

Using the described asymptotic expansions method and the procedure of averaging over a periods of loading and heating for Eqs. (8) and (9), considering the results of similar procedures which led to Eq. (6), the low cycle creep-damage equations can be written in the following form:

$\dot{c}=g_{T}(T) g_{n}\left(M_{k}\right) \frac{\sigma_{i}^{n}}{(1-\omega)^{m}}, \quad c(0)=0$,

$\dot{\omega}=g_{r}\left(M_{k}\right) g_{T}^{\omega}(T) \frac{\sigma^{r}}{(1-\omega)}, \quad \omega(0)=\omega_{0}, \omega\left(t_{*}\right)=\omega_{*}$

where

$$
\begin{gathered}
g_{T}(T)=B \int_{0}^{1} \exp \left(-\frac{Q}{T}\left(1+\sum_{i=1}^{\infty} M_{i}^{T} \sin (2 \pi i \xi)\right)^{-1}\right) d \xi, M_{i}^{T}=\frac{T_{i}^{a}}{T}, \\
g_{T}^{\omega}(T)=D \int_{0}^{1} \exp \left(\frac{-\bar{Q}}{T}\left(1+\sum_{i=1}^{\infty} M_{i}^{T} \sin (2 \pi i \xi)\right)^{-1}\right) d \xi .
\end{gathered}
$$

Now let us consider the case of mono-harmonic sine loading, with frequencies more than $1 \mathrm{~Hz}$

$\bar{\sigma}=\sigma+\sigma^{a} \sin \varpi t$,

where $\sigma^{a}$ and $\sigma$ are the amplitude and the mean stress, respectively.

In this case when the frequencies of oscillations correspond to phenomenon of forced vibrations the processes of dynamic creep as well as the creep-high cycle fatigue interaction can occur [12]. The character of a running processes is different depending of the value of stress cycle asymmetry coefficient $A=\sigma^{a} / \sigma$. The processes of dynamic creep and creep-fatigue interaction are divided by the value of so-called critical stress cycle asymmetry coefficient $A_{c r}$. It is known from experiments [12] that the dynamic creep occurs at low values of $A$, which causes the substantial acceleration of creep strain and damage rates. By the use of the procedures of asymptotic expansions and averaging over a period of forced vibrations like (3) and (4), we obtain dynamic creep averaged 
equations as follows:

$\dot{c}=B(1+H(A)) \frac{(\sigma)^{n}}{(1-\omega)^{m}}, \quad \dot{\omega}=D(1+K(A)) \frac{(\sigma)^{r}}{(1-\omega)^{l}}$

where

$H(A)=\int_{0}^{1}(1+A \sin (2 \pi \xi))^{n} d \xi-1, \quad K(A)=\int_{0}^{1}(1+A \sin (2 \pi \xi))^{r} d \xi-1$.

If the levels of mean stress are low, the value of stress cycle asymmetry coefficient $A$ can be high and exceed the value of $A_{c r}$, which is the known constant for each material. Often $A_{c r} \approx 1$.

In this case of loading the essential high cycle fatigue damage appears, but dynamic creep acceleration become marginal and disappears for high values of $A[12,16]$.

For the description of those phenomena we will use the Yokobori assumption [23] that the total damage increment contains from creep $\omega_{c}$ and high cycle fatigue $\omega_{f}$ parts

$d \omega=d \omega_{c}+d \omega_{f}=F_{c}\left(\sigma, \sigma^{a}, \bar{T}, \omega\right) d t+F_{f}\left(\sigma, \sigma^{a}, \bar{T}, \omega\right) d t$

The creep-damage law (1) and auto-model fatigue-damage law

$d \omega_{F}=\frac{F^{\prime}\left(\sigma^{a}+b \sigma\right)^{p}}{\left(1-\omega_{f}\right)^{q}} d N=\frac{F\left(\sigma^{a}+b \sigma\right)^{p}}{\left(1-\omega_{f}\right)^{q}} d t, \quad N=\frac{t}{T_{F}}$

can be used, where $b$ is a constant, determined for the material under consideration. Often it is taken by assumption that $b=\sigma_{y} / \sigma_{u}$, where $\sigma_{y}$ and $\sigma_{u}$ are respectively the yield stress and tensile strength of a material. Here $F, p, q$ are material constants, and $T_{f}$ is a time to rupture due to high temperature fatigue.

To construct the damage law reproducing the assumption (14) we use the influence functions which reflect the acceleration or retardation of appropriate damage processes. These functions have to be formulated in terms of stress asymmetry coefficient $A$ and its critical value $A_{c r}$ and can be selected in the following form [24]:

$\beta_{f}(A)=1-\exp \left(-\pi \frac{A^{2}}{A_{c r}^{2}}\right), \quad v_{f}(A)=\exp \left(-\pi \frac{A^{2}}{A_{c r}^{2}}\right)$.

The damage law for cyclic loading with frequencies inherent to forced vibrations takes the following form:

$$
\begin{aligned}
& \dot{\omega}=\alpha_{f}(A) D \frac{\sigma^{r}}{(1-\omega)}+\beta_{f}(A) F \frac{\left(\sigma^{a}+b \sigma\right)^{p}}{(1-\omega)^{q}} \\
& \omega(0)=\omega_{0}, \quad \omega\left(t_{*}\right)=\omega_{*}, \quad \alpha_{f}(A)=1+K(A) v_{f}(A) .
\end{aligned}
$$

Now it is necessary to combine the laws derived for slow (10) and (11) and fast variation of loads due to forced vibrations (13) and (16). Schematically the process of complex loading is illustrated in Fig. 1.

The main assumption here is the possibility of coherent application of the averaging procedures. For example, at first we average the creep and damage curves which coincide with slow varying of loading or temperature, e.g., engine's start, work and shutdown. After obtaining these curves in forms (10) and (11) mindful of the fact that forced vibrations do not vanish and we must include them in our equations. This leads to simplificationnow we have to consider the stress law due to results of averaging procedures. Any variation of mean stress changes the values of stress cycle asymmetry coefficient, which leads to possible change of damage law: from dynamic creep to creep-fatigue interaction and vice versa.

So sequential regarding the asymptotical expansions and averaging over the periods of stresses and temperature allow us to construct the universal laws for creep strain and damage parameter rates

$\dot{c}=\frac{B g_{T}(T) g_{n}\left(M_{k}\right)\left(1+H_{n}\left(A_{n}\right) \nu_{f}\left(A_{n}\right)\right) \sigma^{n}}{(1-\omega)^{m}}, c(0)=0$

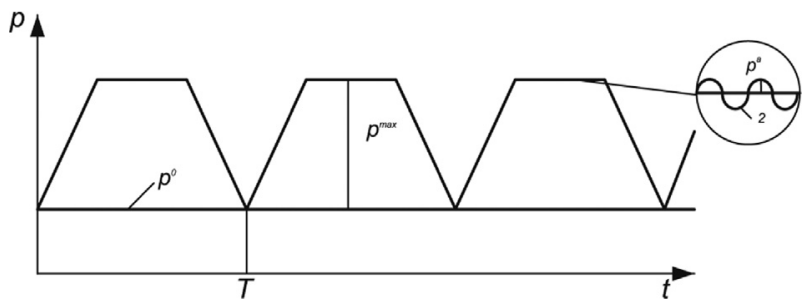

Fig. 1. Schematic illustration of complex cyclic loading.

$$
\begin{aligned}
& \dot{\omega}=D g_{T}(T) g_{r}\left(M_{k}\right) \alpha_{f}\left(A_{r}\right) \frac{\sigma^{r}}{(1-\omega)^{l}}+\beta_{f}\left(A_{r}\right) F \frac{\left(\sigma^{a}+b g_{r}^{1 / r}\left(g_{T}^{\omega}\right)^{1 / r} \sigma\right)^{p}}{(1-\omega)^{q}} \\
& \omega(0)=\omega_{0} ; \omega\left(t_{*}\right)=\omega_{*} \\
& \alpha_{f}(A)=1+K_{r}\left(A_{r}\right) v_{f}\left(A_{r}\right), \\
& \beta_{f}\left(A_{r}\right)=1-\exp \left(-\pi \frac{A_{r}^{2}}{A_{c r}^{2}}\right), v_{f}\left(A_{n}\right)=\exp \left(-\pi \frac{A_{n}^{2}}{A_{c r}^{2}}\right) \\
& g_{n}\left(M_{k}\right)=\int_{0}^{1}\left(1+\sum_{k=1}^{\infty} M_{k} \sin (2 \pi k \xi)\right)^{n} d \xi \\
& g_{r}\left(M_{k}\right)=\int_{0}^{1}\left(1+\sum_{k=1}^{\infty} M_{k} \sin (2 \pi k \xi)\right)^{r} d \xi, M_{k}=\frac{\sigma^{a k}}{\sigma} \\
& g_{T}(T)=\int_{0}^{1} \exp \left(-\frac{Q}{T}\left(1+\sum_{i=1}^{\infty} M_{i}^{T} \sin (2 \pi i \xi)\right)^{-1}\right) d \xi, M_{i}^{T}=\frac{T_{i}^{a}}{T} \\
& g_{T}^{\omega}(T)=\int_{0}^{1} \exp \left(\frac{\left.-\bar{Q}\left(1+\sum_{i=1}^{\infty} M_{i}^{T} \sin (2 \pi i \xi)\right)^{-1}\right) d \xi}{H_{n}=\int_{0}^{1}\left(1+A_{n} \sin (2 \pi \xi)\right)^{n} d \xi-1 ; A_{n}=\frac{A}{g_{r}^{1 / n} g_{T}^{1 / n}},}\right. \\
& K_{r}=\int_{0}^{1}\left(1+A_{r} \sin (2 \pi \xi)\right)^{r} d \xi-1 ; A_{r}=\frac{A}{g_{r}^{1 / r}\left(g_{T}^{\omega}\right)^{1 / r}}
\end{aligned}
$$

Here in order to keep the logic of presentation some notations of influence functions and coefficients are saved, but for this generalized form of creep-damage equations they differ by a constant from above presented.

If we have the material with significant hardening effects in the primary creep stage, all above made considerations can be made for it by similar procedures.

As an example we present the curves describing the creep and long term behavior of specimens made of high temperature nickelbased alloy EI867 (Ni 56-70\%, Fe 4\%, C 0.1\%, Mn 0.3\%, Cr 8.5-10,5\%, Mo $9-11.5 \%$, W 4.3-6\%, Co $4-6 \%$, Al $4.2-4.9 \%$ ) at $T=1173 \mathrm{~K}$. The physical and mechanical properties of the alloy are determined by the use of diagrams of deformation, creep curves and long term data: $E=1.67 \times 10^{5} \mathrm{MPa}, \quad \rho=7.8 \times 10^{3} \mathrm{~kg} / \mathrm{m}^{3}, \quad \nu=0.3, \quad A_{c r}=0.8, \quad n=6.7$, $r=3.92, \quad \mathrm{~B}=2.65 \times 10^{-21} \mathrm{MPa}^{-n} / \mathrm{h}, \quad D=2.4 \times 10^{-13} \mathrm{MPa}^{-m} / \mathrm{h}$, $m=l=7.06, F=4.68 \times 10^{-13} \mathrm{MPa}^{-p} / \mathrm{h}, p=q=3.5$. Golub's experimental data [25] are used, the frequency of cyclic loading is equal to $35 \mathrm{~Hz}$.

The data which are calculated by the use of Eqs. (17) and (18) are compared with the experimental results.

Fig. 2 shows the creep curves. The so-called limit curve, reflecting the correlation between static stress $\sigma$ and amplitude stress $\sigma^{a}$ which lead to fracture at similar time $600 \mathrm{~h}$, is presented in Fig. 3

The data calculated by Eqs. (17) and (18) are given by solid lines. Experimental data obtained by Golub [25] are shown by dots.

Fig. 2 shows the cases of static creep (curve 1, $\sigma=180 \mathrm{MPa}$, $\sigma^{a}=0$; curve $2, \sigma=200 \mathrm{MPa}, \sigma^{a}=0$ ) and cyclic creep with essential interaction of creep and high cycle fatigue damage (curve $\mathbf{3}$, $\sigma=\sigma^{a}=200 \mathrm{MPa}, A=1, A>A_{c r}=0.8$ ). The analysis of curve 3 shows that damage accumulation due to creep and high cycle fatigue 


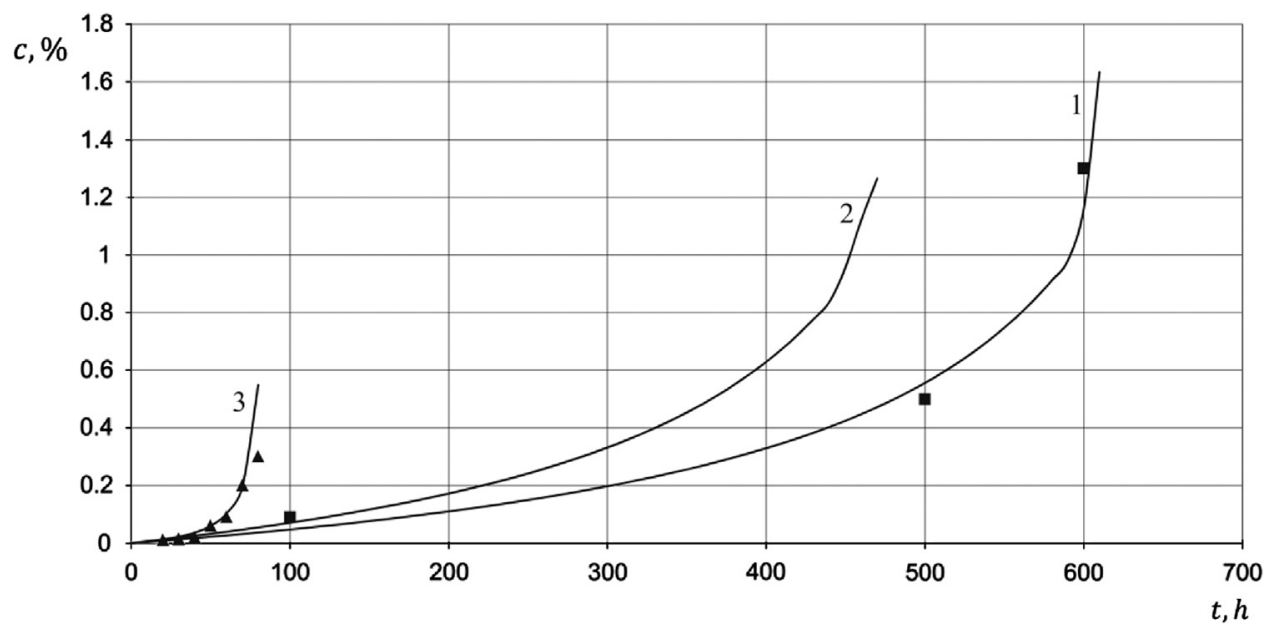

Fig. 2. Creep curves of an high temperature nickel-based alloy EI867 at $T=1173 \mathrm{~K}$.

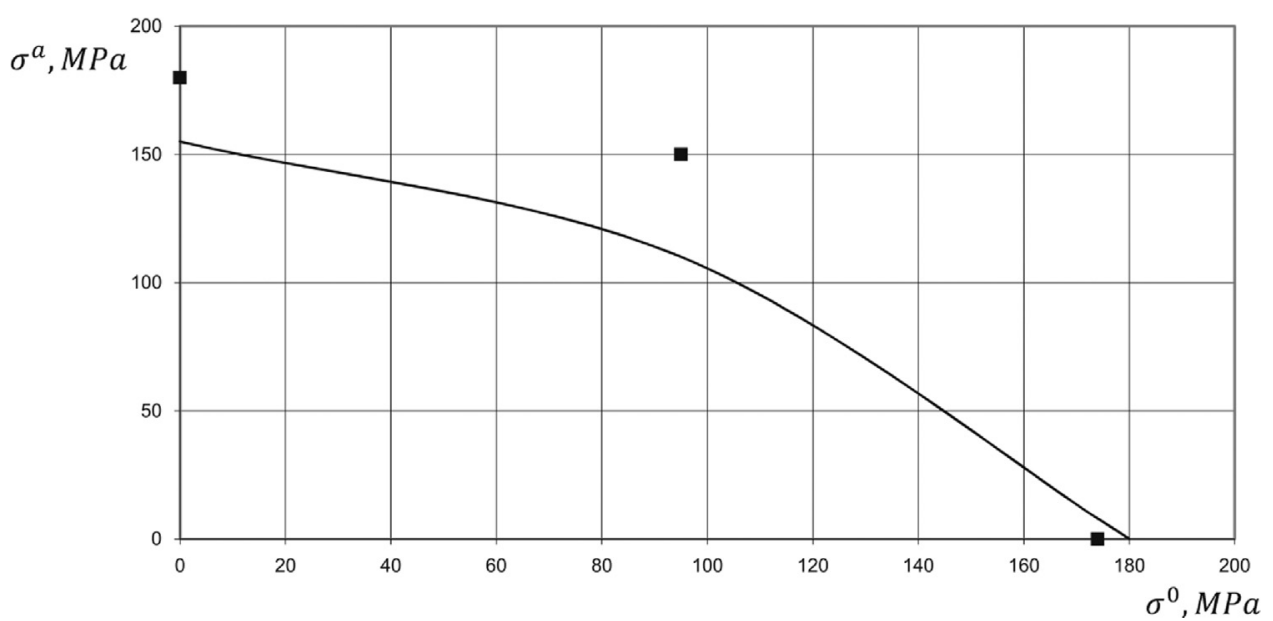

Fig. 3. Limit curves of an high temperature nickel-based alloy El867 at $T=1173 \mathrm{~K}$.

essentially decreases the time to rupture (it is approximately 5 times less in comparison with static loading, curve 2). The rupture strain values are decreased, too.

Comparison of calculated and experimental data for limit curve (Fig. 3) allows us to conclude that damage Eq. (18) correctly reflects the basic fracture laws in wide range of stress cycle asymmetry coefficient: from static loading $(A=0)$ to cyclic creep conditions and pure high cycle fatigue $(A \cong \infty)$.

The distinctions between calculated and experimental data does not exceed $10-28 \%$. The biggest difference of $28 \%$ takes place in more complex case of interaction between creep and high cycle fatigue damage.

The analysis of these results shows that presented state equations describe correctly the influence of additional cyclic loading on the creep strain rate and the time to fracture.

Over the past 25 years the constitutive equations for cyclic loading and heating at creep conditions had been verified on more than 20 materials, including steels, high temperature nickel-based and aluminum alloys at different stresses and temperatures. Three materials were tested at the laboratory of NTU 'KhPI', as well as large amount of published data, like [12,24-29], were analyzed.

Certainly, considered above co-relations can be generalized for the case of complex stress state. It can be done in traditional way by the use of equivalent stresses: von Mises for equation for creep strain rate, for damage equation it has to be taken from fracture criterion [3]. Stress cycle asymmetry coefficients are calculated using the equivalent stresses.

By the use of classical approach for constitutive model formulated for strain rates [22], the final form of constitutive equations for cyclic variation of temperatures and stresses has been obtained as follows:

$\dot{c}_{i j}=\frac{3}{2} \frac{B g_{T}(T) g_{n}\left(M_{k}^{\sigma_{i}}\right)\left(1+H_{n}\left(A_{n}^{i}\right) \nu_{f}\left(A_{n}^{i}\right)\right) \sigma_{i}^{n-1}}{(1-\omega)^{m}} S_{i j}, \quad c_{i j}(0)=0$,

$\dot{\omega}=D g_{T}(T) g_{r}\left(M_{k}^{\sigma_{e}}\right) \alpha_{f}\left(A_{r}^{e}\right) \frac{\sigma_{e}^{r}}{(1-\omega)}+\beta_{f}\left(A_{r}^{e}\right) F \frac{\chi\left(\sigma_{e}^{a}, b g_{r}^{1 / r}\left(g_{T}^{\omega}\right)^{1 / r} \sigma_{e}\right)^{p}}{(1-\omega)^{q}}$

$\omega(0)=\omega_{0}, \omega\left(t_{*}\right)=\omega_{*}, \chi\left(\sigma_{e}^{a}, b \sigma_{e}\right)$ is the expression for equivalent stress for fatigue failure at complex stress state, for example, due to criterion of Sines [30].

$\alpha_{f}(A)=1+K\left(A_{r}^{e}\right) v_{f}\left(A_{r}^{e}\right)$

$g_{n}\left(M_{k}^{\sigma_{i}}\right)=\int_{0}^{1}\left(1+\sum_{k=1}^{\infty} M_{k}^{\sigma_{i}} \sin (2 \pi k \xi)\right)^{n} d \xi, M_{k}^{\sigma_{i}}=\sigma_{i}^{a k} / \sigma_{i}$,

$g_{r}\left(M_{k}^{\sigma_{e}}\right)=\int_{0}^{1}\left(1+\sum_{k=1}^{\infty} M_{k}^{\sigma_{e}} \sin (2 \pi k \xi)\right)^{r} d \xi, M_{k}^{\sigma_{e}}=\sigma_{e}^{a k} / \sigma_{e}$

$\bar{\sigma}=\sigma_{e}\left(1+\sum_{k=1}^{\infty} M_{k}^{\sigma_{e}} \sin \left(\frac{2 \pi k}{T_{\sigma}} t+\gamma_{k}\right)\right), \sigma_{i}=\sqrt{\frac{3}{2} S_{i j} S_{i j}}$ 
$A_{n}^{i}=\frac{A^{i}}{g_{r}^{1 / n} g_{T}^{1 / n}}, A_{r}^{e}=\frac{A^{e}}{g_{r}^{1 / r}\left(g_{T}^{\omega}\right)^{1 / r}}, A^{e}=\frac{\sigma_{e}^{a}}{\sigma_{e}}, \sigma_{i}^{a}=\sqrt{\frac{3}{2} S_{i j}^{a} S_{i j}^{a}}$

$\sigma_{e}=(1-\lambda) \sqrt{\frac{3}{2} S_{i j} S_{i j}}+\lambda \sigma_{I} ; \sigma_{i}^{a k}=\sqrt{\frac{3}{2} S_{i j}^{a k} S_{i j}^{a k}} \sigma_{e}^{a k}=(1-\lambda) \sqrt{\frac{3}{2} S_{i j}^{a k} S_{i j}^{a k}}+\lambda \sigma_{I}^{a k}$,

where $\sigma_{I}, S_{i j}$ are the maximum principal stress and stress deviator components of tensor $\sigma_{i j}$; $\lambda$ is the parameter of material's sensitivity for the type of fracture; $S_{i j}^{a}$ and $S_{i j}^{a k}$ are the deviator components of amplitude stress tensors $\sigma_{i j}^{a}$ and $\sigma_{i j}^{a k}$.

Temperature influence functions $g_{T}(T), g_{T}^{\omega}(T)$ are the same as in (18).

Finally let us note that although constructed constitutive model (19) and damage kinetic Eq. (20) look complicated, the practical use of them is simple.

Now let us describe the material constants which are included in constitutive Eqs. (19)-(21). They can be organized in four groups. First one includes six creep-damage constants $B, n, k, D$, $r$, and $l$, whose values have to be determined by the use of creep curves at static loading by three values of stresses at fixed temperature $T_{1}$. These constants are conventional for BaileyNorton-Rabotnov-Kachanov Eq. (1).

Second group includes two constants $Q$ and $\bar{Q}$ for state equations of type (8) and (9) and reflects the difference between the temperatures $T_{1}$ and $T_{2}$ in creep and damage rates. For this step we need the experimental results and constants from the first group (obtained at the temperature $T_{1}$ ) and at least two creep curves at temperature $T_{2}$.

For the case of forced vibrations imposed on the static loading, the constants of the third group have to be determined. They include three constants $F, p$, and $q$, which are obtained from the high cycle fatigue long term strength diagram; one constant $b$, reflects the influence of static loading on fatigue damage accumulation, and $A_{c r}-$ the so-called critical value of stress cycle asymmetry parameter, which divides the processes of dynamic creep acceleration and pure high cycle fatigue imposed on the static stress. $A_{c r}$ is usually within the range $0.8<A_{c r}<1.1$, so, if it is impossible to carry out the dynamic creep experiment, its value can be accepted as $A_{c r}=1$.

Frequently the material behavior can be characterized by smaller number of constants at creep or fatigue conditions: $(n=k, r=l)$ or $(k=l)$ for creep-damage processes and $(p=q)$ at high cycle fatigue damage. In such a case the third stress level in experiments is used for validation of obtained state equations.

The last constant $\lambda$ is used in the long-term criterion and varies within the following ranges: $0<\lambda<1$ [3].

So the total number of constants is 14 . This number is ultimate and is required for the hypothetic case of temperature variation, slow and fast variation of stresses in a wide range, which causes both creep damage and high cycle fatigue damage. It is very important to stress here that research engineers do not have to conduct any additional experiments on cyclic loading of material specimens. All the tests are standard, except the test for determination of $A_{c r}$, but the constitutive Eqs. (19)-(21) can be used assuming that $A_{c r}=1$ as mentioned above.

Validation of the particular parts of Eqs. (19)-(21) for complex stress state had been done $[16,18,19,24,26-28,31]$. In addition, the thermodynamic justification of those equations was performed.

Satisfactory agreement allows us to propose the generalized form of the creep-damage state equations at cyclic loading and heating.

We have obtained quasi-static equations reflecting basic phenomena connected with influence of cyclic loading with different frequencies on the creep-damage process. Certainly, the proposed form of constitutive equations needs to be further verified for different materials and combinations of cyclic loading's programs, but presented result allows us to simplify their use in particular cases.

\section{General problem statement for cyclic creep-damage problems for shells of revolution}

Let us present general mathematical problem statement for boundary-initial value creep problem. We regard the solid isotropic body with volume $V$ which is fixed in a surface part $S_{1}$. The body is loaded by volume forces $f_{i}$, the traction $p_{i}$ acts on the surface part $S_{2}$. The case of constant in time volume forces is regarded: $f=f\left(x_{i}\right), x_{i} \in V, i=1,2,3$. Traction has constant value $p_{i}^{(0)}=p_{i}^{(0)}\left(x_{i}\right)$ and varies in a time through the cycle

$$
\begin{aligned}
& \Phi_{i}(t): p_{i}=p_{i}^{(0)}+\Phi_{i}(t), \quad x \in S_{2}, \\
& \Phi_{i}(t)=p_{i}^{\max } \sum_{k=1}^{\infty} Z_{k} \sin \left(\Omega_{k} t+\beta_{k}\right)+p_{i}^{a} \sin (\Omega t),
\end{aligned}
$$

where $p_{i}^{\max }, p_{i}^{a}$ are the amplitudes of appropriate components of traction; $Z_{k}=\sqrt{a_{k}^{2}+b_{k}^{2}} ; \Omega_{k}=2 \pi k / T ; \Omega=2 \pi / T_{2}$.

Considering the above made assumptions, we present mathematical problem statement for creep problem by set of equations as follows:

$$
\begin{aligned}
& \varepsilon_{i j}=e_{i j}+c_{i j}+\varepsilon_{i j}^{T}, \quad \sigma_{i j, j}+f_{i}=\rho \frac{\partial^{2} u_{i}}{\partial t^{2}}, \quad \varepsilon_{i j}=\frac{u_{i, j}+u_{j, i}+u_{k, i} u_{k, j}}{2}, \quad x_{i} \in V ; \\
& \sigma_{i j}=D_{i j k l}\left(\varepsilon_{k l}-c_{k l}-\varepsilon_{k l}^{T}\right) ; \quad \sigma_{i j} n_{j}=p_{i}^{(0)}+\Phi_{i}(t), \quad x_{i} \in S_{2} ; \\
& u_{i}=\bar{u}_{i}, \quad x_{i} \in S_{1} ; \quad u_{i}\left(x_{i}, 0\right)=c_{i j}\left(x_{i}, 0\right)=0
\end{aligned}
$$

where $n_{j}$ are the components of the unit vector which is normal to body's surface, $j=1,2,3$; $\{D\}$ is a tensor of elastic properties of the material; $u_{i}$ are known values of the displacements on the surface $S_{1}$, which are constant in a time; and $\rho$ is the material density.

Total strain consists of elastic $e_{i j}$, temperature $\varepsilon_{i j}^{T}$ and creep $c_{i j}$ components.

Cyclic variation of temperatures is determined from the nonstationary heat conduction problem:

$a_{T}^{2} \Delta \bar{T}+Q_{T}=\dot{\bar{T}}, a_{T}^{2}=k_{T} /\left(c_{T} \rho\right), \quad Q_{T}=F /\left(c_{T} \rho\right)$

where $\Delta$ is Laplace's operator; $c_{t}, k_{t}$, are respectively the coefficients of specific heat and heat conduction; and $F$ is the density of thermal sources.

To solve system (22) we use the asymptotic expansions method with subsequent averaging over a period of cyclic loading, similar to the approach used for constitutive equations. The use of these methods allows us to divide the system of Eq. (22) into two. First system arises from expressions which result from averaging and describes the processes which are run in slow basic motion [16]; the second is obtained by subtraction from (22) the first system. These systems are not independent, they are connected by Eqs. (19)-(21).

So in order to determine the stress-strain state of creeping body subjected to cyclic traction, we have to solve the following first system of equations, where all unknowns vary in slow time scale:

$$
\begin{aligned}
& \sigma_{i j, j}+f_{i}=0 \quad x_{i} \in V ; \quad \sigma_{i j} n_{j}=p_{i}, \quad x_{i} \in S_{2} ; \\
& \varepsilon_{i j}=C_{i j k l} \sigma_{k l}+c_{i j}+\varepsilon_{i j}^{T}, \quad \varepsilon_{i j}=\frac{1}{2}\left(u_{i, j}+u_{j, i}+u_{k, i} u_{k, j}\right), \quad x_{i} \in V ; \\
& u_{i}=\bar{u}_{i}, \quad x_{i} \in S_{1} ; \quad u_{i}\left(x_{i}, 0\right)=c_{i j}\left(x_{i}, 0\right)=0 .
\end{aligned}
$$

Constitutive Eqs. (19)-(21) have to be added. Components of amplitude stress tensors which are included in this constitutive model are determined from another systems, formulated for fast 
varying time scale $(k=1,2, \ldots \ldots)[18]$ :

$\sigma_{i j, j}^{a k}=-\rho\left(\Omega_{k}\right)^{2} u_{i}^{a k}, \quad x_{i} \in V ; \quad \sigma_{i j}^{a k} n_{j}=p_{i}^{\max } Z_{k}, x_{i} \in S_{2}$,

$\varepsilon_{i j}^{a k}=\frac{1}{2}\left(u_{i, j}^{a k}+u_{j, i}^{a k}\right)=C_{i j m n} \sigma_{m n}^{a k}, \quad x_{i} \in V ; u_{i}^{a k}=0, x_{i} \in S_{1}$;

and

$\sigma_{i j, j}^{a}=-\rho(\Omega)^{2} u_{i}^{a}, \quad x_{i} \in V ; \quad \sigma_{i j}^{a} n_{j}=p_{i}^{a}, \quad x_{i} \in S_{2}$,

$\varepsilon_{i j}^{a}=\frac{1}{2}\left(u_{i, j}^{a}+u_{j, i}^{a}\right)=C_{i j m n} \sigma_{m n}^{a}, \quad x_{i} \in V ; \quad u_{i}^{a k}=0, \quad x_{i} \in S_{1}$;

By solving systems (25) and (26) for each harmonic components $k$ the values of amplitude stress tensors are determined. They are used in constitutive model (19)-(21).

Thereby the solution of creep-damage problem in the case of cyclic loading is divided into to two systems. Basic system (24) has to be solved jointly with Eqs. (19)-(21) where amplitude stress components are determined from auxiliary systems (25) and (26).

Obtained results are now applied for modeling of cyclic behavior of thin shells in creep conditions. Shells of revolution are considered. We limit ourselves by the case of homogeneous temperature field at each time moment, e.g., the effects of heating and cooling are neglected. Owing to subsequent using of Finite Element Method (FEM) let us pass to variation formulation of the problem. Instead of equilibrium equations in (24) the Lagrange functional is used

$$
\begin{aligned}
& \int_{S}\left(f_{i j k l} \varepsilon_{i j}^{m} \delta \varepsilon_{i j}^{m}-g_{i j k l} \chi_{k l} \delta \chi_{i j}\right) d S-\int_{S} p_{q} \delta u_{q} d S-\int_{S} N_{i j}^{c} \delta \varepsilon_{i j}^{m} d S \\
& \quad+\int_{S} M_{i j}^{c} \delta \chi_{i j} d S=0
\end{aligned}
$$

where $\delta \varepsilon_{i j}^{m}$ are the variations of total strains in the middle surface of shell; $\delta \chi_{i j}$ are the variations of changes in curvature; $\delta u_{q}$ are the variations of displacements vector, $q=1,2,3$; and $\delta_{i j}$ is Kronecker delta.

$f_{i j k l}=\frac{E \delta}{1-\nu^{2}}\left(\delta_{i k} \delta_{j l} \frac{1-\nu}{2}+\nu \delta_{i j} \delta_{k l}\right) ; \quad g_{i j k l}=\frac{E \delta^{3}}{12\left(1-\nu^{2}\right)}\left(\delta_{i k} \delta_{j l} \frac{1-\nu}{2}+\nu \delta_{i j} \delta_{k l}\right)$.

Shell forces and moments are as follows:

$$
\begin{aligned}
N_{11}^{c}= & \frac{E}{1-\nu^{2}} \int_{-\delta / 2}^{\delta / 2}\left(c_{11}+\nu c_{22}\right) d z, \\
& N_{22}^{c}=\frac{E}{1-\nu^{2}} \int_{-\delta / 2}^{\delta / 2}\left(c_{22}+\nu c_{11}\right) d z, S^{c}=\frac{E}{2(1+\nu)} \int_{-\delta / 2}^{\delta / 2} c_{12} d z ; \\
M_{11}^{c}= & \frac{E}{1-\nu^{2}} \int_{-\delta / 2}^{\delta / 2}\left(c_{11}+\nu c_{22}\right) z d z, \\
& M_{22}^{c}=\frac{E}{1-\nu^{2}} \int_{-\delta / 2}^{\delta / 2}\left(c_{22}+\nu c_{11}\right) z d z ; \quad H^{c}=\frac{E}{2(1+\nu)} \int_{-\delta / 2}^{\delta / 2} c_{12} z d z .
\end{aligned}
$$

The finite element of conical shell with four nodes was used for calculations. Each node has seven degrees of freedom: three displacements and four angles of rotation. Using the standard FEM procedures [32] allows us to reduce the variational equality (27) to the system of linear algebraic equations

$[K]\{U\}=\left\{P^{v}\right\}+\left\{P^{c}\right\}+\left\{P^{p}\right\}+\left\{P^{n}\right\}$,

where $[K]$ is global matrix of stiffness; $\{U\}$ is the vector of nodal displacements; $\left\{P^{\nu}\right\}$ is the generalized vector of external forces; $\left\{P^{c}\right\}$ is the generalized vector of nodal forces caused by creep irreversible strains; $\left\{P^{p}\right\}$ is the generalized vector of nodal forces originated by projections of generalized forces on normal vector; and $\left\{P^{n}\right\}$ is the generalized vector of nodal forces caused by nonlinear component of elastic strains.

System (29) has to be solved at each time step. Time integration methods, like predictor-corrector of 3rd order and Euler method, are used.

We use the solutions as follows:

$\left([K]-\Omega_{k}^{2}[M]\right)\left\{\Delta^{a k}\right\}=\left\{P^{a k}\right\}, \quad\left([K]-\Omega^{2}[M]\right)\left\{\Delta^{a}\right\}=\left\{P^{a}\right\}$ which are FEM formulations of systems (25) and (26) for the determination of amplitude values of stresses for each harmonic components. It is necessary to calculate the current values of influence functions in constitutive Eqs. (19) and (20). Here $[M]$ is the mass matrix of the system; $\Omega_{k}, \Omega$ are the frequencies of external loading; $\Delta^{a k}, \Delta^{a}$ are the vectors of amplitude values at poly-harmonic loading through a cycle and at forced vibrations, correspondingly.

So the system (29) is used for the analysis of creep-damage processes in shell structures. At arbitrary time step the vector of nodal displacements is determined as a solution of this system. This vector is used for determination of stresses, strains, damage parameter in each finite element.

After achieving by the damage parameter its critical value $\omega_{*} \leq 1$ the process of hidden damage accumulation is regarded as completed. This time value determines the long term strength of the structure or its certain part. The place of the initiation of first macro-crack is determined by the place of the finite element with maximal value of damage parameter $\omega_{*}$.

The described method has been implemented in computer code with pre- and post-processing programs [18] in the National Technical University "KhPI" (Ukraine). As an example the results of the investigations of different cyclic creep-damage behaviors of the conical panel are presented.

\section{Cyclic creep and damage in conical shell}

In order to analyze the laws of deformation and failure due to cyclic loading at creep conditions let us regard the open conical shell made of high temperature nickel-based alloy EI867 at $T=1173$ (Fig. 4).

The shell's length is $L=8 \mathrm{~m}$, smaller radius is $R=1.5 \mathrm{~m}$, bigger radius is $R=2 \mathrm{~m}$, thickness is $h=0.01 \mathrm{~m}$. Shell edges are clamped.

The shell is subjected to internal pressure which has slow and fast varying parts

$p(t)=p^{0}+p^{\max }\left(\frac{2}{3}-\sum_{k=1}^{\infty} \frac{4}{\pi^{2} k^{2}} \cos \left(\frac{2 \pi k}{T} t\right)\right)+p^{a} \sin \left(2 \pi f_{2} t\right)$

Pressure static component is $p^{0}=1.2 \mathrm{MPa}$, amplitude value is $p^{\max }=0.6 \mathrm{MPa}$. Amplitude of fast varying pressure is $p^{a}=0.25 p^{0}$. The rectangular cycle of stress slow varying is studied, the value of period is equal to $1 h$, wherein $p^{\max }=0$ each $50 \mathrm{~min}$. Frequency of oscillations is $f_{2}=0.1 f$. Here $f$ is the first eigenfrequency of the shell. Its value which was found by solving the eigenvalue problem by Jacoby method, is $f=342 \mathrm{~Hz}$.

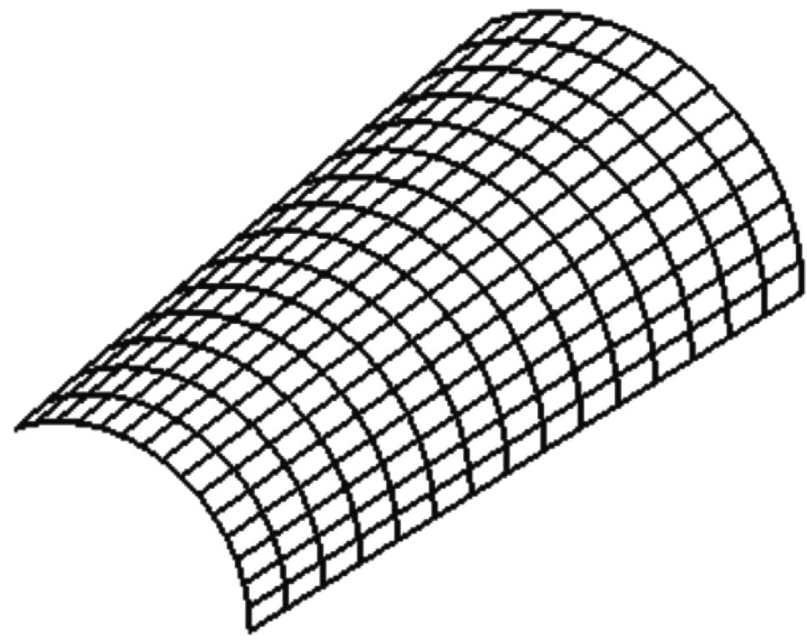

Fig. 4. FE mesh of open conical shell. 


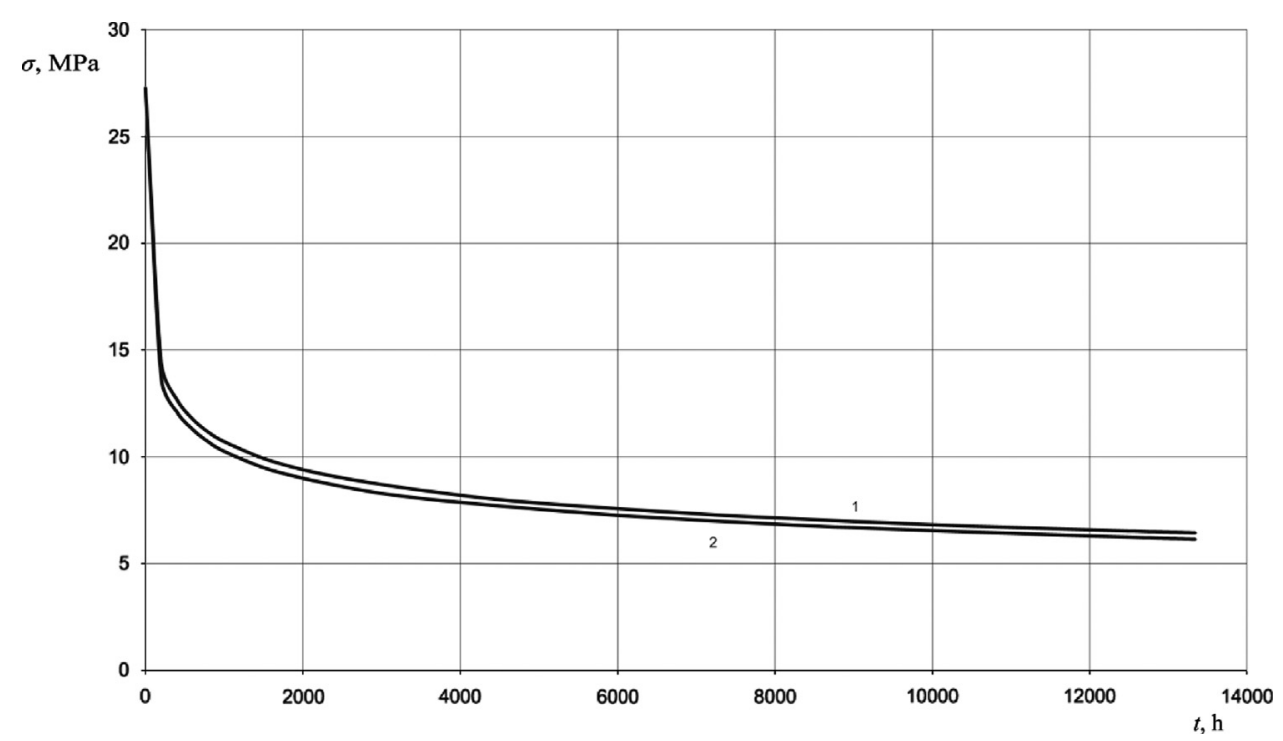

Fig. 5. Von Mises equivalent stress relaxation at the finite element where fracture begins.

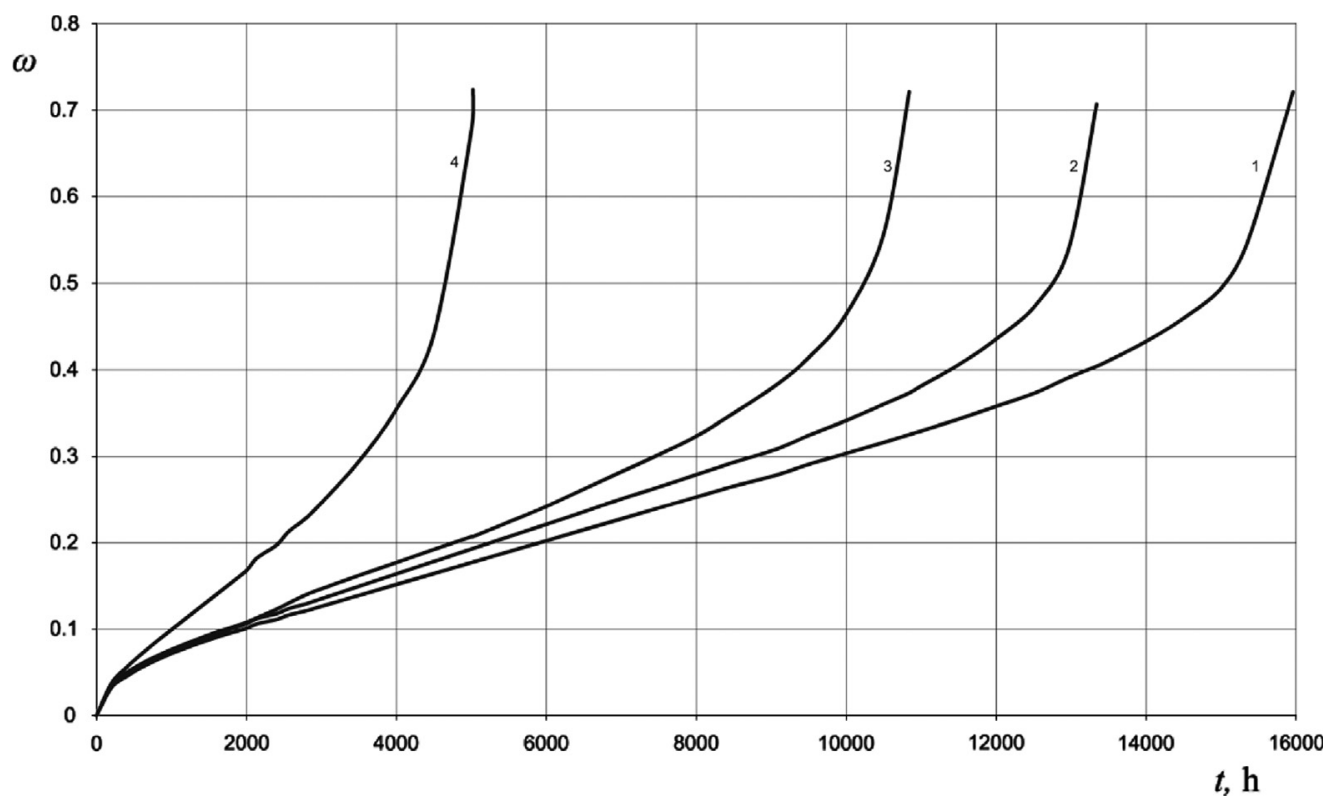

Fig. 6. Growth of the damage parameter at the finite element where fracture begins.

Four problems of cyclic loading of the shell under consideration were analyzed. Let us number them: pure static loading with pressure $p_{0}, \mathbf{1}$; dynamic creep with only fast oscillating pressure component, $p^{\max }=0,2$; cyclic creep with pressure varying due to the law (31), 3; cyclic creep with pressure varying due to law (31) with consideration of creep-high cycle fatigue damage interaction, 4. The values of time to fracture for these problems substantially differ: $1-16,000 \mathrm{~h}, 2-13,340 \mathrm{~h}, 3-10,840 \mathrm{~h}$, and $4-5020 \mathrm{~h}$.

Fig. 5 shows the graphical representation of stress relaxation in a point of outer surface in the central cross-section, where the fracture occurs further $\left(\omega=\omega_{*}=0.72\right)$. Fig. 6 illustrates the growth of the damage parameter's values in this point. The curve number coincides with the number of the problem. The final deformed state of the shell in the 4th problem is characterized by maximum deflection equal to $0.439 \mathrm{~mm}$. Other problems have similar displacements distribution and differ insignificantly: for example, creep at static loading (problem 1) runs slower, but needs more time for fracture start, wherein the maximum final deflection is practically the same and is equal to $0.438 \mathrm{~mm}$.

Fig. 6 shows the difference in damage growth for different cyclic programs. We can see that supposition of any cyclic loading accelerates damage accumulation. The time to fracture at dynamic creep (2) is smaller than in the case of pure static loading. Consideration of rectangular slow cycles of loading/unloading (3) and especially creep-fatigue interaction accelerates the damage accumulation process so that fracture time decreases significantly (more than three times for problem $\mathbf{4}$ ).

Comparing the results of problems $\mathbf{2}$ and $\mathbf{3}$ we can observe essential role of fatigue damage component in area, where fracture occurs even for problems, where at the starting moment of cyclic loading stress cycle asymmetry coefficient is less than $A_{c r}$. Equivalent stress significantly relaxes here from the beginning of creep process until fracture moment: it decreases approximately five times (Fig. 5). In this connection stress cycle asymmetry coefficient 
increases to be more than $A_{c r}$ and influence of fatigue damage contribution becomes prevailing.

\section{Conclusions}

The method of solution for creep-damage problems of structural elements subjected to cyclic loading at high temperatures is presented in this paper. Proposed method of solution and constitutive equations allow us to consider different types of cyclic behavior in one and the same manner. Analysis of the results for conical shell shows that cyclic loading can essentially accelerate the growth processes of strains and damage parameters as well as stress relaxation. Due to stress relaxation the role of high temperature fatigue damage in the global process of damage accumulation at creep conditions is important and may become principal.

One of the simplest and time-tested the Rabotnov-Kachanov phenomenological model [3] with one scalar damage parameter is suggested in the paper in order to demonstrate the possibilities of the asymptotic and averaging methods for deriving the cyclic creep-damage constitutive equations. This simple but very useful model is not based on the Physics of Metals data, which establishes the mechanisms of creep running accompanied by hidden damage accumulation due to voids growth [33-35], for example. Mostly it was made intentionally, to demonstrate the approaches for constructing the creep-damage constitutive equations for the case of cyclic loading or heating.

The main goal of this paper is to discuss the derivation method and hence the cyclic loading problem is given only as an illustrative example. The authors do not expect that suggested theory will be frequently used for the most common case of cyclic loading, presented in the paper, but the presented derivation method can be used in more sophisticated problems. It is very difficult to predict all possible variations of loading, heating, cyclic changing of different parameters etc., so it is important to have the general procedure. Nevertheless, presented particular cases, for example for combinations of static and cyclic loading or cyclic heating will be useful for engineers. Just because of this, the discussion of the problem is built by inductive way, from the case of one assumption to several.

In addition, let us note that the proposed approach with a scalar damage parameter is applicable for the tensor damage models of cyclic loading/heating. For example, for the description of dynamic creep processes in duralumin plates it was implemented with the tensor damage model [36].

In the same way the proposed approach can be applied for widely used creep-damage mechanism-based equations for steels and alloys [33-35] because of the fact that basic qualitative laws for creep-damage processes are well-known. Generally these laws describe the increase of creep strain and damage accumulation rates at cyclic loading or heating under fixed value of static loading as well as appropriate decreasing of time to fracture. The asymptotic methods and the methods of averaging over a cycle period can be used for any constitutive equations for creep strain, hardening as well as for one or several different damage parameters (like proposed in [33]) regardless of the method of their derivation.

As for the question of the generality of the proposed procedures and their comparison with the others, we can stress, that the advantages of the asymptotic approaches will be mostly exhibited in processes with large numbers of cycles such as in the problem of superposition of forced oscillations on the constant static loading, which occurs, for example, under operating conditions of turbine casing, rotors and blades. To our knowledge, the analysis of the complex stress state is possible only by using the discussed procedures, because direct integration from one cycle to another is practically impossible due to very large numbers of cycles $\left(10^{5}-10^{7}\right)$. The accumulating computational errors in direct approach, as well as the physical effects of dynamic creep-damage acceleration, which have to be reflected in constitutive equations, give rise to the application of our procedure.

Regarding the low-cycle processes, one can argue that advantages of the proposed procedures can be whittled away by the use of direct integration method or limit analysis. However, this opinion, which bases on the experience with widespread cyclic plasticity problems, is wrong: even 100 cycles of slow varying of loading or heating in creep conditions leads to subsequent acceleration of creep strain rate and damage accumulation (see [29], e.g., Fig. 6). That is why it is necessary to derive the appropriate constitutive equations as well as to use the solution of boundaryinitial value problem, obtained by asymptotic procedures and averaging over a period. The number of hundreds of cycles with the value of small parameter of 0.01 can be regarded as a lowerbound estimate here.

For small number of cycles (from one-two up to several tens) the method is inapplicable. From the mathematical point of view, we do not have a small parameter in this case. Mechanical analysis of this case shows that fracture is possible here only due to action of large stresses, which overcome the yield limit. In this case the classical well tested methods [1-3] have to be used. The proposed procedures currently cannot be used for limit analysis, shakedown and in presence of the plastic strains in general. These problems will be studied in future.

In conclusion the last potential application of proposed generalized equations and the method of their solving have to be highlighted. Creep-damage processes are essentially non-linear, so simple consequent analysis of different influences of cyclic loading and heating will be incorrect (for instance, even in modern studies the influence of the creep acceleration due to forced vibrations of the structure is neglected). Research engineers require an instrument for global cyclic creep-damage process estimation, even though this instrument has certain limitations. It is important to be able to compare different design solutions at the stage of numerical simulation. The present paper is an attempt to propose such instrument.

\section{References}

[1] R.D. Campbell, Creep-fatigue correlation for 304 stainless steel subjected to strain controlled cycling with hold times as peak strain, ASME Pap. 71 (6) (1971) 887-892

[2] D.R. Hayhurst, Estimates of creep rupture lives of structures subjected to cyclic loading, Int. J. Mech. Sci. 18 (1976) 75-83.

[3] J. Lemaitre, J.-L. Chaboche, Mechanics of Solid Materials, University Press, Cambridge, 1994.

[4] R. Viswanathan, Damage Mechanisms and Life Assessment of HighTemperature Components, ASM International, Metals Park, Ohio, 1995.

[5] B. Dambrine, J.P. Mascarell, Designing turbine blades for fatigue and creep, Def. Sci. J. 38 (4) (1988) 413-429.

[6] F.P.E. Dunne, D.R. Hayhurst, Modelling of combined high-temperature creep and cyclic plasticity in components using continuum damage mechanics. Proc. R. Soc. Lond.: Math. Phys. Sci. 437 (1901) (1992) 567-589.

[7] H.D. Chandler, S. Kwofie, A description of cyclic creep under conditions of axial cyclic and mean stresses, Int. J. Fatigue 27 (5) (2005) 541-545.

[8] T. Yamamoto, M. Iwata, M. Sakane, Y. Tsukada, H. Nishimura, Effect of strain waveform on creep-fatigue life for Sn-8Zn-3Bi solder, Fatigue Fract. Eng. Mater. Struct. 30 (5) (2007) 376-386.

[9] S. Amiable, A computational lifetime prediction of a thermal shock experiment. Part I: thermomechanical modelling and lifetime prediction, Fatigue Fract. Eng. Mater. Struct. 29 (3) (2006) 175-182.

[10] S. Amiable, A computational lifetime prediction of a thermal shock experiment. Part II: discussion on difference fatigue criteria, Fatigue Fract. Eng. Mater. Struct. 29 (3) (2006) 219-227.

[11] J.T. Boyle, J. Spence, Stress Analysis for Creep, Butterworth Publishers, Southampton (1983) 283.

[12] S. Taira, R. Ohtani, Theory of High Temperature Strength of Material, Metallurgia, Moscow, 1986 (in Russian, translated from Japanese).

[13] M. Nagode, High cycle thermo-mechanical fatigue: damage operator approach, Fatigue Fract. Eng. Mater. Struct. 32 (6) (2009) 505-514. 
[14] K. Watashi, Creep-fatigue failure test and analysis of a vessel-type structure subjected to cyclic thermal transients, Nucl. Eng. Des. 140 (3) (1993) 349-372.

[15] J.P. Cusumano, A. Chatterjee, Steps towards a qualitative dynamics of damage evolution, Int. J. Solids Struct. 37 (44) (2000) 6397-6417.

[16] D. Breslavsky, O. Morachkovsky, A new model of nonlinear dynamic creep, in: Proceedings of the IUTAM Symposium on Anisotropy, Inhomogenity and Nonlinearity in Solid Mechanics, Kluwer Academic Publishers, Dordrecht, 1995, pp. 61-166.

[17] H. Altenbach, D. Breslavsky, O. Morachkovsky, K. Naumenko, Cyclic creepdamage in thin-walled structures, J. Strain Anal. Eng. Des. 35 (1) (2000) 1-11.

[18] D. Breslavsky, O. Morachkovsky, O. Tatarinova, High temperature creep and long term strength of structural elements under cyclic loading, Strength Mater. 40 (5) (2008) 531-537.

[19] D.V. Breslavs'kyi, Y.M. Korutko, O.K. Morachkovs'kyi, Cyclic thermal creep model for the bodies of revolution, Strength Mater. 43 (2) (2011) 134-143.

[20] Y.N. Rabotnov, Creep Problems in Structural Members, North Holland, Amsterdam, 1969.

[21] N.N. Moiseev, Asymptotic Methods of the Non-Linear Science, Nauka, Moscow, 1981 (in Russian)

[22] J.A. Sanders, F. Verhulst, Averaging Methods in Nonlinear Dynamical Systems, Springer-Verlag, New York, 1985.

[23] T. Yokobori, Physics and Mechanics of Fracture and Strength of Solids, Mettallurgia, Moscow, 1971 (in Russian, translated from Japanese).

[24] D. Breslavsky, O. Morachkovsky, Cyclic creep constitutive equations with consideration of creep-fatigue interaction, in: Proceedings of the 1st International Conference on Mechanics of Time Dependent Materials, SEM, Bethel, 1995, pp. 61-66.

[25] V.P. Golub, Cyclic creep of high temperature nickel-based alloys, Naukova Dumka, Kiev, 1983 (in Russian).

[26] D. Breslavsky, O. Morachkovsky, Dynamic creep modelling with coupled creep and fatigue damage: computational and experimental results for structures, creep and coupled processes, in: Proceedings of the 5th International Symposium on Creep and Coupled Processes (Selected and Revised Papers),
Bialowieza, 1995, Bialystok Technical University Publisher, Bialystok, 1996, pp. 141-146.

[27] D. Breslavsky, O. Morachkovsky, New experiments in dynamic creep, in: Proceedings of the 15th Symposium on Experimental Mechanics of Solids, Warsaw Technical University, Warsaw, 1992, pp. 29-31.

[28] D.V. Breslavsky, O.K. Morachkovsky, S.A. Shipulin, Creep and rupture of notched plates under fast cyclic load, in: Proceedings of the 17th Symposium on Experimental Mechanics of Solids, Warsaw Technical University, Warsaw, 1996, pp. 118-123.

[29] G.I. Guarnieri, The creep-rupture properties of aircraft sheet alloys subjected to intermittent load and temperature, ASTM, Spec. Tech. Publ. 165 (1954) 105-146.

[30] G. Sines, G. Ohgi, Fatigue criteria under combined stresses or strains, Trans. ASME. J. Eng. Mater. Technol. 103 (4) (1981) 82-90.

[31] D. Breslavsky, Y. Korytko, O. Morachkovsky, High temperature creep and damage accumulation in cyclically loaded axisymmetrical bodies of revolution, in: Nonlinear Dynamics-2010: Proceedings of the Third International Conference, NTU “KhPI", Kharkov, 21-24 September, 2010, pp. 467-472.

[32] K.J. Bathe, Finite-Elemente Methoden, Heidelberg: Springer-Verlag, Berlin, 1990.

[33] Z.L. Kowalewski, D.R. Hayhurst, B.F. Dyson, Mechanism-based creep constitutive equations for an aluminium alloy, J. Strain Anal. 29 (1994) 309-316.

[34] K. Naumenko, H. Altenbach, A. Kutschke, A combined model for hardening, softening, and damage processes in advanced heat resistant steels at elevated temperatures, Int. J. Damage Mech. 20 (2011) 578-597.

[35] H. Altenbach, S. Kozhar, K. Naumenko, Modelling creep-damage of an aluminum-silicon eutectic alloy, Int. J. Damage Mech. 22 (5) (2012) 683-698.

[36] D. Breslavsky, Y. Korytko, V. Mietielov, O. Morachkovsky, O. Tatarinova, Numerical calculations of creep damage at cyclic loading by use of tensor damage parameter model, in: Nonlinear Dynamics-2013: Proceedings of the Fourth International Conference, Sevastopol, Kharkov, June 19-22, 2013, pp. 379-384. 